\title{
Review of four major biomolecular target sites for COVID-19 and possible inhibitors as treatment interventions
}

\author{
Bright Vigbedor ${ }^{1 *}$, Clement Okraku Tettey ${ }^{2}$, Edward Ken Essuman ${ }^{3}$, Isaac Kyere ${ }^{4}$, Albert Aniagyei ${ }^{1}$, Nii Korley Kortei ${ }^{3}$, \\ Adjoa Agyemang Boakye ${ }^{2}$, Jonathan Osei-Owusu ${ }^{5}$ \\ ${ }^{1}$ Department of Basic Sciences, School of Basic and Biomedical Sciences, University of Health and Allied Sciences, Ho, Ghana, West Africa. \\ ${ }^{2}$ Department of Biomedical Sciences, School of Basic and Biomedical Sciences, University of Health and Allied Sciences, Ho, Ghana, West Africa \\ ${ }^{3}$ Department of Nutrition and Dietetics, School of Allied Health Sciences, University of Health and Allied Sciences, Ho, Ghana, West Africa. \\ ${ }^{4}$ Department of Chemistry, Kwame Nkrumah University of Science and Technology, Ghana. \\ ${ }^{5}$ Department of Biological, Physical and Mathematical Sciences, University of Environment and Sustainable Development, Somanya, Ghana
}

\begin{tabular}{l}
\hline ARTICLE INFO \\
\hline Received on: 15/01/2021 \\
Accepted on: 12/04/2021 \\
Available online: 05/08/2021 \\
\\
\hline Key words: \\
COVID-19, pandemic, ACE2, \\
drug, chloroquine, inhibitor.
\end{tabular}

\begin{abstract}
This paper focuses on the review of major target sites in both the host organism and the severe acute respiratory syndrome coronavirus 2 virus and the inhibitors that have been screened so far to unravel possible treatment agents. In this review, four major target sites were found to be the main sites where the design of possible inhibitors and treatment interventions could be probed. The four major sites that were reviewed include main protease, transmembrane protease, serine 2, RNA-dependent RNA polymerase, and angiotensin-converting enzyme 2. Several existing drug candidates have been screened as inhibitors of the reviewed target sites and could serve as lead agents, prodrugs, and prospects for the treatment of coronavirus disease 2019. In this review, several inhibitors such as chloroquine (CQ) and hydroxychloroquine have gone through the clinical trial phase and are being utilized for the management of the disease. Drug candidates such as CQ, derivatives, remdesivir, and favipiravir have been used for the treatment of infected persons with $100 \%$ recovery rate. It is, therefore, imperative that further structural activity relationship and modifications be carried out using these drug candidates as models for synthesizing new analogues as treatment options.
\end{abstract}

\section{INTRODUCTION}

Severe acute respiratory-coronavirus 2019 (SARSCoV-19) is an infectious disease caused by the Corona subfamily Orthocoronavirinae with Coronaviridae as the family. It belongs to the order Nidovirales and realm Riboviria represented as an enveloped virus with a single-stranded RNA genome and nucleocapsid of helical symmetry (Li et al., 2020). The disease has been officially labeled as a pandemic by the World Health Organization and its outbreak has plagued the world with severe economic losses and crippling of world health systems. As a

\footnotetext{
"Corresponding Author

Bright Vigbedor, Department of Basic Sciences, School of Basic and Biomedical Sciences, University of Health and Allied Sciences, Ho, Ghana,West Africa.E-mail: ybvigbedor@uhas.edu.gh
}

result, several interventions have been explored to help curtail the situation (World Health Organization, 2020).

The epicenter of the deadly pandemic, Wuhan, and its transmission across the entire globe has brought debilitating effects on the World Health systems (Zhou et al., 2020). Even though vaccine development has taken the center stage in the fight against the coronavirus disease 2019 (COVID-19), the development process is fraught with several challenges such as late registration of harmful side effects. Nevertheless, the possibility of exploring several options in the combat of the disease is eminent (Kaufmann et al., 2014; Prajapati and Vashistha, 2011; Stratton et al., 2011).

From a statistical point of view, about 178,635,780 cases of COVID-19 have been recorded across the globe with $3,867,850$ deaths and 163,156,628 recoveries as at June, 2021 [WHO, 2021]. Amid the uncertainties in the fight against the disease, the approach to tackling the pandemic has been through the four main strategies, preventive measures, drug discovery, 
vaccine development, and symptomatic treatment. However, in the area of drug discovery, several agents have been explored for their efficacy against various groups of the COVID-19. Based on lessons learned from severe acute respiratory syndrome and middle east respiratory syndrome outbreaks, lack of drugs capable of pan-coronavirus antiviral activity increases the vulnerability of public health systems to a highly pathogenic coronavirus pandemic (Totura and Bavari, 2019).

The objective of this review document is to explore the various mechanisms implicated in the mode of infection as well as the life cycle of the virus and to find possible drug candidates as inhibitors of these mechanistic pathways.

\section{STRUCTURAL BIOLOGY AND MODE OF INFECTION}

The severe acute respiratory syndrome coronavirus 2 (SARS-CoV-2) virion is $50-200 \mathrm{~nm}$ in diameter with four structural proteins involved in its metabolic activities and sustenance (Wong et al., 2020). The four proteins involved are as follows: the spike (S) is the structural protein manifested in the form of spikes on its spherical outer membrane and its involvement in the attachment of the virus onto the host cell surface (Zhang et al., 2020). The nucleocapsid protein $(\mathrm{N})$ is the carrier of the RNA genome, whereas the envelope (E) and the membrane protein (M), as well as the spike proteins, serve as encapsulation and envelope for the viral nuclear constituents (Zhang et al., 2020, Zhu et al., 2020). Biomolecular representation of SARS-CoV-2 is shown in Figure 1.

The mode of infection of the COVID-19 virus is linked to its affinity for the main enzyme, angiotensin-converting enzyme 2 (ACE2) as the virus attaches itself to body surfaces such as lungs, arteries, heart, kidney and intestines which are the site availability of the enzyme. As the virus infects the surfaces of these tissues, RNAdependent polymerases are activated to induce replication of the virus (Hoffmann et al., 2020; Letko et al., 2020; Zhou et al., 2020).

\section{TARGET SITES AND DRUG CANDIDATES FOR COVID-19}

In the advent of the outbreak, several scientists have sought to review various classes of drugs based on their mechanism

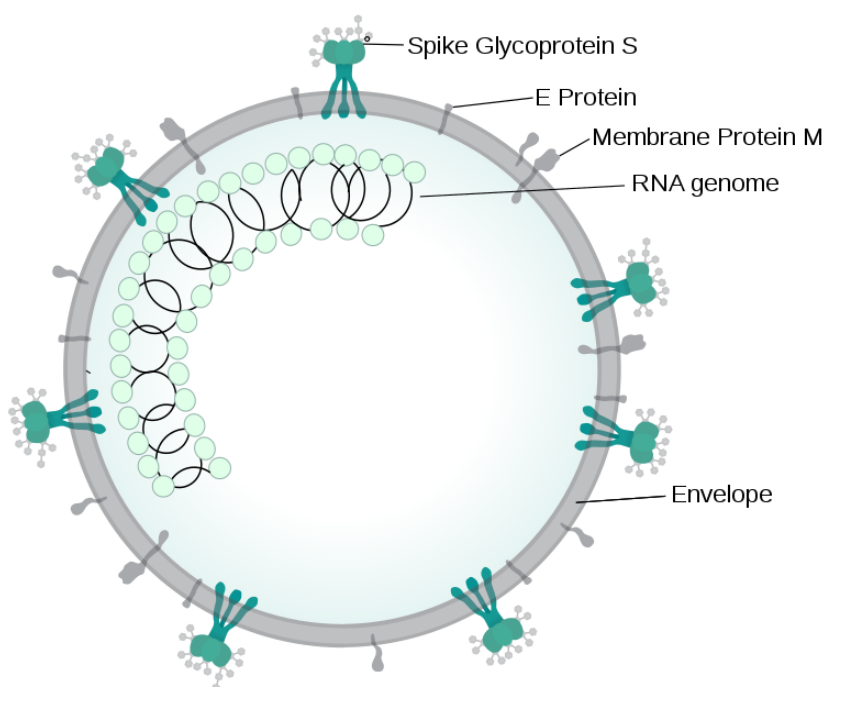

Figure 1. Biomolecular representation of SARS-CoV-19. of action for clinical trial protocols and have screened them to find suitable lead agents for immediate treatment of infected persons. Given the mode and mechanism of action of COVID-19 and review of current antiviral agents as antidotes to combating the multiplying synthesizing machinery, drugs have been reviewed by laying much emphasis on the various routes of actions. It is, therefore, prudent to review agents based on their effects on hydrolyzing or cleaving the RNA polymeric chain to curtail replication. Several drug screening strategies have been employed to select possible drug candidates based on their structural motifs or pharmacophore.

Other routes of attack such as the enzymes involved in the synthesis of the protein involved in the survival of the virus could be targeted. Given this, lots of lead agents could be screened to inhibit the enzymes involved in their action, for example, agents that restrain the synthesis works by hindering a protein known as RNA-dependent RNA polymerase. This polymerase is utilized by numerous RNA viruses including coronaviruses to replicate themselves (Cheng et al., 2005). On the other hand, other RNA viruses that are known as retroviruses (HIV) use an enzyme called reverse transcriptase. This subsequently creates DNA from an RNA blueprint. This implies that the antiviral drug compound remdesivir (RDV) has significant potential to be effective since coronaviruses also use RNA-dependent enzymes (Cheng et al., 2005).

\section{Angiotensin-converting enzyme 2 (ACE2) target}

Several drug screening protocols have been sought to regulate the activity of ACE2 as a treatment intervention to reduce the severity of infection by the virus. The enzyme is attached to the outer surface of cells in the lungs, heart, kidney and intestines and it is used as an attachment protein by the spike protein of the virus. Design and screening of prospective inhibitors of the enzyme could help decrease the levels of ACE2 and further reduce infection [Donoghue et al., 2000; Hamming et al., 2004; National Center for Biotechnology Information (NCBI), 2020].

Available data are covering theoretical, experimental, preclinical, and clinical evidence of the effectiveness of chloroquine (CQ) and hydroxylchloroquine (HCQ) for the people infected with COVID-19. The mechanism of action from literature studies on how CQ has been applied for several strains of viruses involves its ability to disrupt metabolic activities in cells by altering the $\mathrm{pH}$ of their vesicles to halt the replication of RNA. The pathway, therefore, disrupts its protein synthesis and hence cell death. The activities of CQ and its derivative, HCQ, have been tested in vitro (Liu et al., 2020). According to Liu et al.'s (2020) study, both CQ and HCQ are frail bases that are known to raise the $\mathrm{pH}$ of acidic intracellular organelles, such as endosomes/lysosomes, necessary for layer fusion. Also, CQ could inhibit severe acute respiratory syndrome coronavirus entry through changing the glycosylation of ACE2 receptor and spike protein. Time-of-addition experiment confirmed that HCQ effectively inhibited the entry step, as well as the postentry stages of SARS-CoV-2, which was also found upon CQ treatment. Structural examples of ACE2 inhibitors are represented in Figure 2.

Based on this information, Gurwitz (2020) suggested using available angiotensin receptor 1 blockers, such as losartan, as therapeutics for reducing the severity of COVID-19 infections. 
(c)

Chloroquine

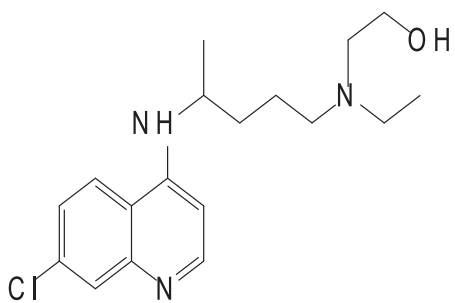

Hydroxychloroquine

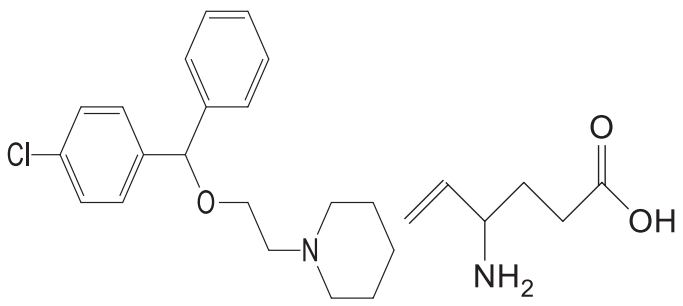

Figure 2. Structural examples of ACE2 inhibitors.

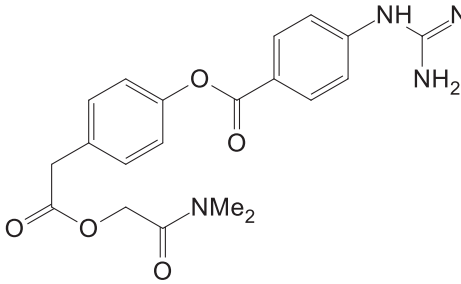

Camostat

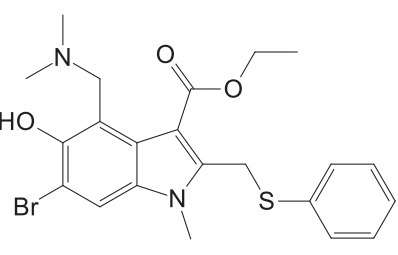

Umifernovir
Figure 3. Structural examples of TMPRSS2 inhibitors.

\section{Transmembrane protease, serine 2 , and transmembrane} serine protease 2 inhibitors

Transmembrane protease, serine 2 (TMPRSS2) enzyme is very vital for the attachment of the spike protein to the ACE2 of host cell surfaces. The SARS-CoV-2 after attachment to the host cell surface releases the protease enzyme TMPRSS2 which then cuts open the spike protein to release the fusion peptide protein to firmly anchor itself onto the host cell. The RNA of the virus is then released onto the host cell to support the replication and production of copies of itself which subsequently infect more cells (Hoffmann et al., 2020; Wu et al., 2020).

The screening of several synthetic and natural products against these enzymes could also constitute an enhanced treatment option against the virus. Drugs such as camostat, umifenovir, and imatinib have been screened as potent inhibitors of TMPRSS2 (Hoffmann et al., 2020; Jiansheng et al., 2020). Figure 3 shows some examples of TMPRSS2 inhibitors.

\section{RNA-dependent RNA polymerase (RdRp) inhibitors}

The RdRPs, also called RNA replicates, catalyze the replication of RNA from an RNA template and are essential proteins encoded in the genomes of all RNA-containing viruses with no DNA stage. Several drug candidates have been found to inhibit the replication of RNA by blocking replication of flaviviruses, filoviruses, bunyaviruses, arenaviruses, noroviruses,<smiles>N#C[C@]1(c2ccc3c(N)ncnn23)O[C@H](P)[C@@H](O)[C@H]1O</smiles>

Remdeivir<smiles>NC(=O)c1nc(F)cnc1O</smiles>

Favipiravir
Figure 4. Structural examples of RNA polymerase inhibitors.

and other RNA viruses (Dong et al., 2020). Drug candidates such as favipiravir are converted to the phosphorylated form (favipiravirRTP) in cells which are recognized as a substrate by viral RNA polymerases. This inhibits RNA polymerase activity. Because of this mechanism pathway, drug candidates such as favipiravir, RDV, and darunavir have yielded improved clinical conditions of infected patients (Delang et al., 2018). The clinical trial potential of the drug was tested alongside other antiviral drugs such as lopinavir/ ritonavir with superior potency and fewer adverse reactions (Furuta et al., 2017). Furthermore, the triphosphate derivative of RDV has also been found to inhibit the RNA replication of the virus by the incorporation of itself into the growing RNA chain of MERS coronavirus RNA-dependent RNA polymerase (MERSCoV-RdRp). The effect of RDV on the replication of the virus has been the target for the screening of several available lead agents as inhibitors of MERS-CoV-RdRp. Figure 4 shows the structural examples of RNA polymerase inhibitors.Given this mechanism, several other agents have been screened through drug screening in silico and an enzyme activity test to give more options in terms of evaluating possible drug candidates for SARS-CoV-19. The agents that have been screened include indinavir, saquinavir, lopinavir, carfilzomib, ritonavir, RDV, atazanavir, darunavir, tipranavir, fosamprenavir, enzaplatovir, presatovir, abacavir, bortezomib, elvitegravir, maribavir, raltegravir, montelukast, deoxyrhapontin, polydatin, chalcone, disulfiram, carmofur, shikonin, ebselen, tideglusib, PX12, TDZD-8, cyclosporin A, and cinanserin (Dong et al., 2020).

\section{Main protease (Mpro)}

The Mpro has been established through experimental studies as the main enzyme involved in the processing of the polyproteins involved in the translation of COVID-19 and the RNA-dependent RNA polymerase, which aids in the catalysis of the virus RNA replication. The enzyme has therefore been considered as a possible target for the screening of inhibitors (Verma et al., 2020). The crystal structure of the enzyme has been established and deposited in the protein database for public access (Liu et al., 2020). The utilization of the target site has led to the evaluation of several drug candidates such as nelfinavir, pitavastatin, perampanel, and praziquantel through molecular docking studies with nelfinavir as the best candidate after the studies (Wu et al., 2020).

Other interventions in the quest to identifying agents that would inhibit the replication of the virus involve the surface 


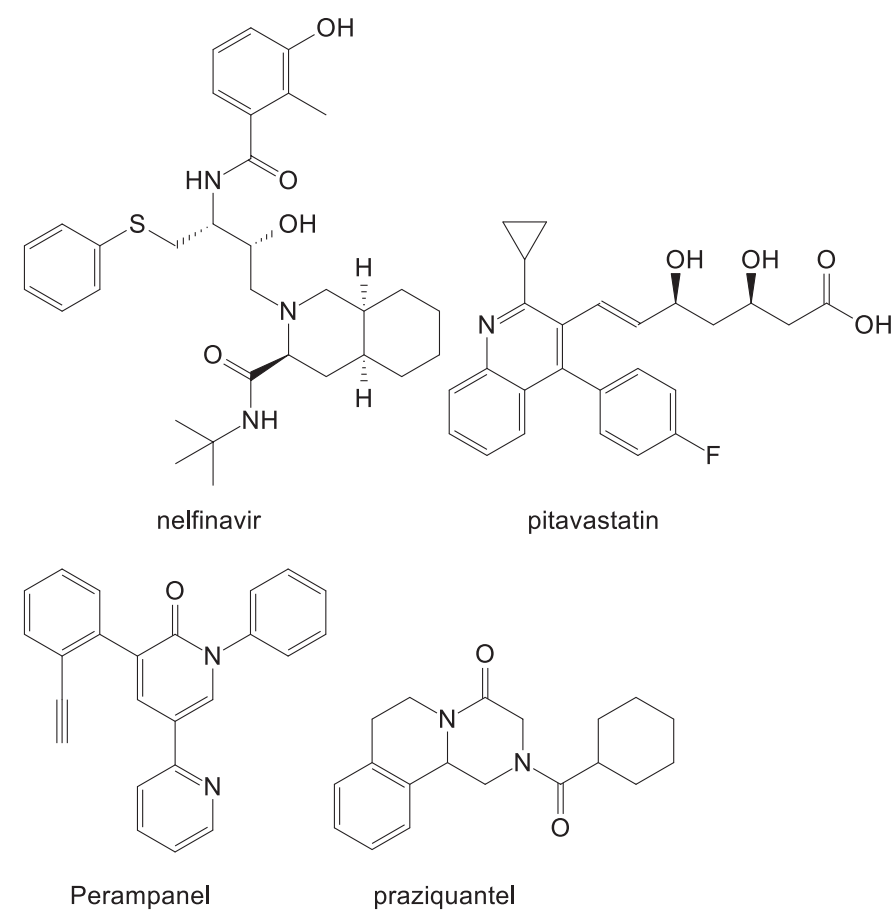

Figure 5. Structural examples of Mpro inhibitors (Ghosh et al., 2021).

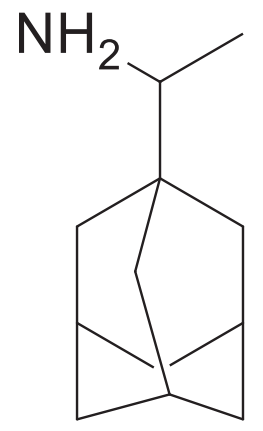

Figure 6. Structure of rimantadine.

binding potentials of rimantadine to nucleocapsid of SARS-CoV-2 using AutoDock Vina in chimera and discovery Studio software for the ligand-drug interaction studies. It was established that rimantadine binds to the nucleocapsid protein with a binding potential of 7.5 and hence could be considered in clinical trials studies. The compound has the structural feature of a primary tricyclic amine (Fig. 6) and hence structural activity studies could be carried out on the binding potential of derivatives of it.

\section{CONCLUSION AND RECOMMENDATION}

In conclusion, several existing drugs are effective in vitro or in vivo against the novel coronavirus at therapeutic doses. The efficacy of such drugs could be enhanced by structural activity relationship studies. This review study offers a repository of drug candidates and their mechanism of action to carry out a series of protocols to understand their actions. This will aid in designing novel compounds with a higher degree of efficacy. In the review, drug candidates such as CQ, hydroxychloroquine, umifenovir, favipiravir, RDV, oseltamivir, thalidomide, and thymosin have gone through clinical trials studies for various degree of severity of the disease. Due to the novel nature of the disease, it is prudent to observe preventive measures as well as focusing on eliminating the virus for good. The advent of screening of small synthetic molecules as well as natural products as lead agents is imminent. Other protocols for appropriate policy formulation to tackle the issue of boosting the immune system to help combat pandemic are imperative. This calls for the urgent integration of teams of experts from the scientific community and political, regional, and world economic associations such as the AU, EU, ECOWAS, US, World Bank, IMF, and United Nations to develop a policy framework to help regulate ethics governing research into the risk factors associated with the design of biochemical molecules.

\section{AUTHORS' CONTRIBUTIONS}

Vigbedor, B.Y. drafted the manuscript and was in charge of the supervision. Tettey, C.O., Essuman, E. K., and Kortei, N.K. drafted the manuscript. Osei-Owusu, J., Aniagyei, A., Kyere, I., and Boakye, A. A. conducted a critical revision of the manuscript.

\section{CONFLICT OF INTEREST}

The authors report no financial or any other conflicts of interest in this work.

\section{FUNDING}

The authors received no funding for the study.

\section{ETHICAL APPROVALS}

Not Applicable.

\section{PUBLISHER'S NOTE}

This journal remains neutral with regard to jurisdictional claims in published institutional affiliation.

\section{REFERENCES}

Cheng A, Zhang W, Xie Y, Jiang W, Arnold E, Sarafianos SG, Ding J. Expression, purification, and characterization of SARS coronavirus RNA polymerase. Virol, 2005; 335(2):165-76.

Delang L, Abdelnabi R, Neyts J. Favipiravir as a potential countermeasure against neglected and emerging RNA viruses. Antiviral Res, 2018; 153:85-94.

Donoghue M, Hsieh F, Baronas E, Godbout K, Gosselin M, Stagliano N, Donovan M, Woolf B, Robison K, Jeyaseelan R, Breitbart RE. A novel angiotensin-converting enzyme-related carboxypeptidase (ACE2) converts angiotensin I to angiotensin 1-9. Circ Res, 2000; 87(5):e1-9.

Furuta Y, Komeno T, Nakamura T. Favipiravir (T-705), a broadspectrum inhibitor of viral RNA polymerase. Proc Japan Acad B, 2017; 93(7):449-63.

Ghosh K, Amin SA, Gayen S, Jha T. Chemical-informatics approach to COVID-19 drug discovery: Exploration of important fragments and data mining-based prediction of some hits from natural origins as main protease (Mpro) inhibitors. Journal of Molecular Structure. 2021 Jan 15; 1224:129026.

Gurwitz D. Angiotensin receptor blockers as tentative SARSCoV-2 therapeutics. Drug Dev Res, 2020; 81(5):537-40.

Hamming I, Timens W, Bulthuis ML, Lely AT, Navis GV, van Goor H. Tissue distribution of ACE2 protein, the functional receptor for SARS coronavirus. A first step in understanding SARS pathogenesis. J Pathol, 2004; 203(2):631-7. 
Hoffmann M, Kleine-Weber H, Schroeder S, Krüger N, Herrler T, Erichsen S, Schiergens TS, Herrler G, Wu NH, Nitsche A, Müller MA. SARS-CoV-2 cell entry depends on ACE2 and TMPRSS2 and is blocked by a clinically proven protease inhibitor. Cell, 2020; 181:1-10.

Kaufmann SH, McElrath MJ, Lewis DJ, Del Giudice G. Challenges and responses in human vaccine development. Curr Opin Immunol, 2014; 28:18-26.

Letko M, Marzi A, Munster V. Functional assessment of cell entry and receptor usage for SARS-CoV-2 and other lineage B betacoronaviruses. Nat Microbiol, 2020; 5(4):562-9.

Li H, Liu SM, Yu XH, Tang SL, Tang CK. Coronavirus disease 2019 (COVID-19): current status and future perspective. Int J Antimicrob Agents, 2020; 55(5):105951.

Liu J, Cao R, Xu M, Wang X, Zhang H, Hu H, Li Y, Hu Z, Zhong W, Wang M. Hydroxychloroquine, a less toxic derivative of chloroquine, is effective in inhibiting SARS-CoV-2 infection in vitro. Cell Discov, 2020; 6(1):1-4.

National Center for Biotechnology Information (NCBI). ACE2, angiotensin I converting enzyme 2. U.S. National Library of Medicine, Bethesda, MD, 2020. Available via https://www.ncbi.nlm.nih.gov/ gene $? \mathrm{Db}=$ gene $\& \mathrm{Cmd}=$ DetailsSearch\&Term $=59272$

National Health Commission (NHC). Outbreak notification of the People's Republic of China. Phylogeny of SARS-like beta coronaviruses, next train. National Health Commission (NHC), Beijing, China, 2020.

Prajapati BS, Vashishtha VM. Adverse events following immunization (AEFI), Vaccine Safety, and Misinformation against Vaccination. In Vashishtha VM, Kalra A, Thacker N (eds.). FAQs on vaccines and immunization practices. Jaypee Brothers, Medical Publishers Pvt. Limited, Delhi, India, p 64, 2011.

Stratton K, Ford A, Rusch E, Clayton EW, Committee to Review Adverse Effects of Vaccines, Institute of Medicine. Adverse effects of vaccines: evidence and causality. National Academies Press, Washington, DC, 2011

Totura AL, Bavari S. Broad-spectrum coronavirus antiviral drug discovery. Expert Opin Drug Dispos, 2019; 14(4):397-412.
Verma D, Kapoor S, Das S, Thakur K. Potential inhibitors of SARS-CoV-2 main protease (Mpro) identified from the library of FDA approved drugs using molecular docking studies. Preprints, 2020; 2020040149; https://doi.org/10.20944/preprints202004.0149.v1

WHO (2021). Covid19 global situation. Available at: https:// covid19.who.int/ (Accessed :18/06/2021).

Wong MC, Cregeen SJ, Ajami NJ, Petrosino JF. Evidence of recombination in coronaviruses implicating pangolin origins of nCoV-2019. BioRxiv, 2020.

World Health Organization. Coronavirus disease 2019 (COVID-19): situation report, 72. World Health Organization, Geneva, Switzerland, 2020.

Wu C, Liu Y, Yang Y, Zhang P, Zhong W, Wang Y, Wang Q, Xu Y, Li M, Li X, Zheng M. Analysis of therapeutic targets for SARS-CoV-2 and discovery of potential drugs by computational methods. Act Pharm Sin B, 2020; 10(5):766-88.

Zhang T, Wu Q, Zhang Z. Probable pangolin origin of SARS-CoV-2 associated with the COVID-19 outbreak. Curr Biol, 2020; 30(7):1346-51.e2.

Zhou P, Yang XL, Wang XG, Hu B, Zhang L, Zhang W, Si HR, Zhu Y, Li B, Huang CL, Chen HD. A pneumonia outbreak associated with a new coronavirus of probable bat origin. Nature, 2020; 579(7798):270-3.

Zhu N, Zhang D, Wang W, Li X, Yang B, Song J, Zhao X, Huang B, Shi W, Lu R, Niu P. A novel coronavirus from patients with pneumonia in China, 2019. N Engl J Med, 2020; 382(8):727-33.

\section{How to cite this article:}

Vigbedor B, Tettey CO, Essuman EK, Kyere I, Aniagyei A, Kortei NK, Boakye AA, Osei-Owusu J. Review of four major biomolecular target sites for COVID-19 and possible inhibitors as treatment interventions. J Appl Pharm Sci, 2021; 11(08):192-196. 